\title{
Correction to: RGD4C peptide mediates anti-p21Ras scFv entry into tumor cells and produces an inhibitory effect on the human colon cancer cell line SW480
}

Chen-Chen Huang ${ }^{1,2+}$, Fang-Rui Liu ${ }^{1 \dagger}$, Qiang Feng ${ }^{2}$, Xin-Yan Pan² ${ }^{2}$ Shu-Ling Song ${ }^{2}$ and Ju-Lun Yang ${ }^{1,2^{*}}$

\section{Correction to: BMC Cancer 21, 321 (2021)}

https://doi.org/10.1186/s12885-021-08056-4

Following publication of the original article [1], the authors reported a typesetting error. Figures 4 and 5 were transposed. The correct Figs. 4 and 5 are supplied below and the original article [1] has been corrected.

* Correspondence: yangjulun@sina.com

${ }^{\dagger}$ Chen-Chen Huang and Fang-Rui Liu contributed equally to this work. ${ }^{1}$ School of Medicine, Kunming University of Science and Technology, 727

South Jing Ming Road, Chenggong County, Kunming 650500, Yunnan Province, China

${ }^{2}$ Department of Pathology, 920th Hospital of Joint Logistics Support Force of PLA, 212Daguan Rd, Xishan District, Kunming 650032, Yunnan Province,

China

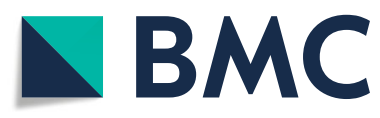

(- The Author(s). 2021 Open Access This article is licensed under a Creative Commons Attribution 4.0 International License, which permits use, sharing, adaptation, distribution and reproduction in any medium or format, as long as you give appropriate credit to the original author(s) and the source, provide a link to the Creative Commons licence, and indicate if changes were made. The images or other third party material in this article are included in the article's Creative Commons licence, unless indicated otherwise in a credit line to the material. If material is not included in the article's Creative Commons licence and your intended use is not permitted by statutory regulation or exceeds the permitted use, you will need to obtain permission directly from the copyright holder. To view a copy of this licence, visit http://creativecommons.org/licenses/by/4.0/. The Creative Commons Public Domain Dedication waiver (http://creativecommons.org/publicdomain/zero/1.0/) applies to the data made available in this article, unless otherwise stated in a credit line to the data. 


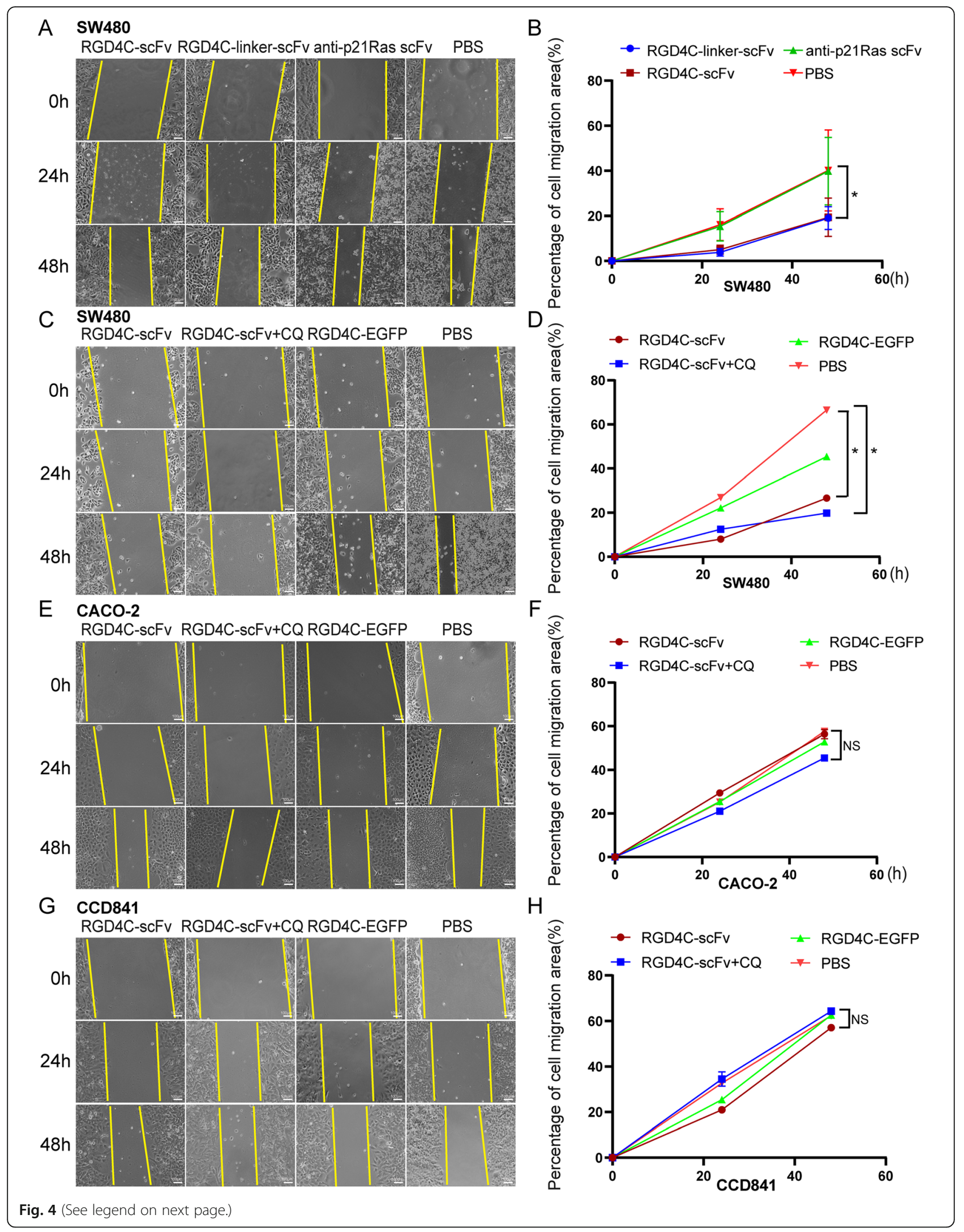


(See figure on previous page.)

Fig. 4 The antitumor efficacy of RGD4C-p21Ras-scFv in vitro. $\mathbf{a}$ and $\mathbf{b}$ Cell migration was measured with a scratch test after SW480 cells were cocultured with $20 \mu \mathrm{M}$ RGD4C-scFv, RGD4C-linker-scFv or the anti-p21Ras scFv for $0 \mathrm{~h}, 24 \mathrm{~h}$, and $48 \mathrm{~h}$. The migration of SW480 cells was inhibited in the RGD4C-scFv and RGD4C-linker-scFv groups compared with the anti-p21 Ras scFv and PBS control groups. $\mathbf{c}$ and $\mathbf{d}$ The migration of SW480 cells was inhibited in the RGD4C-scFv and RGD4C-scFv+CQ groups compared with RGD4C-EGFP and PBS groups. Moreover, the migration inhibition effect of RGD4C-scFv+CQ groups was higher than RGD4C-scFv group. $\mathbf{e}-\mathbf{h}$ There were no difference the migration of CACO-2 and CCD841 cells in the RGD4C-sCFv and RGD4C-scFv+CQ groups compared with RGD4C-EGFP and PBS groups 

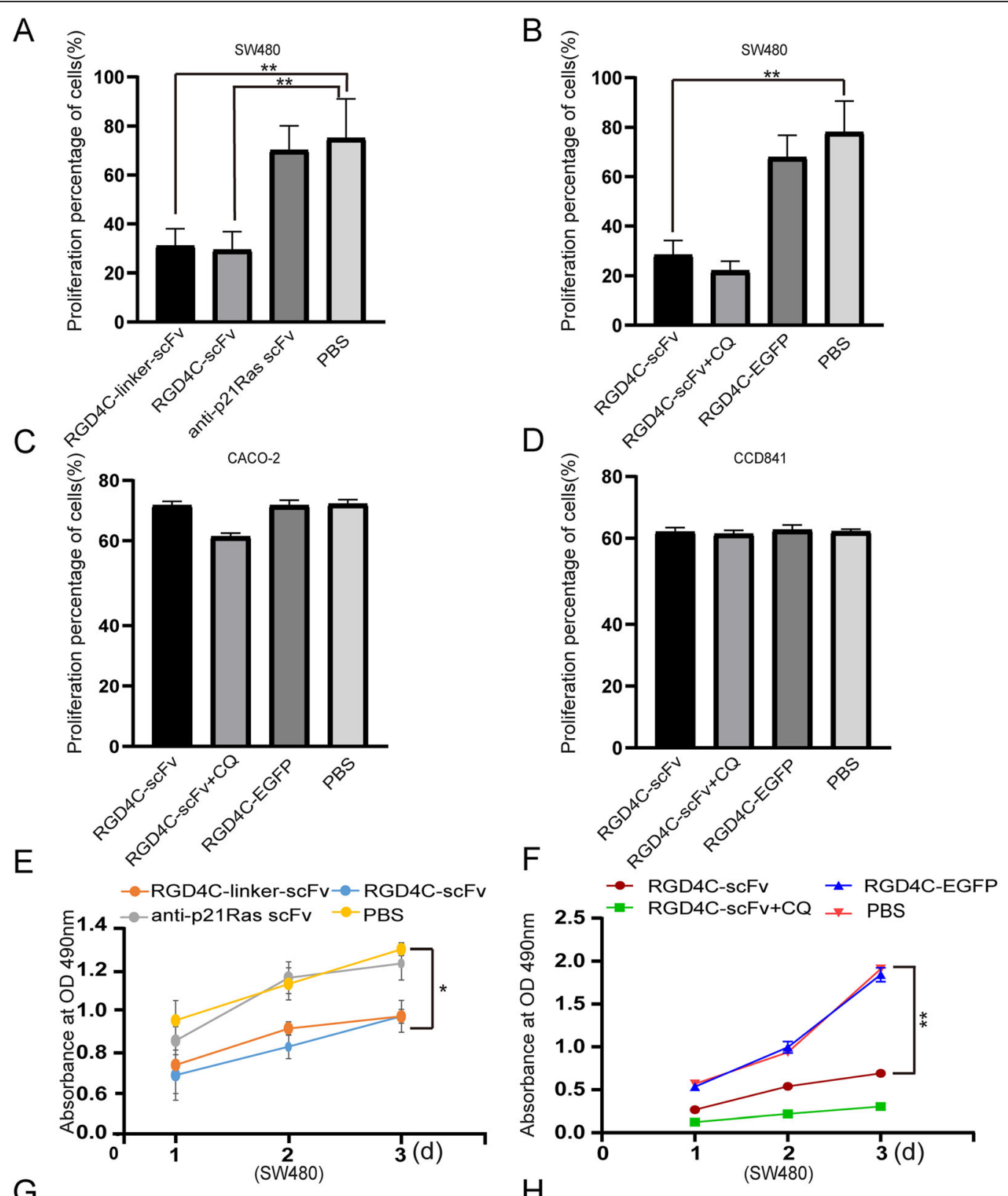

G

$\mathrm{H}$
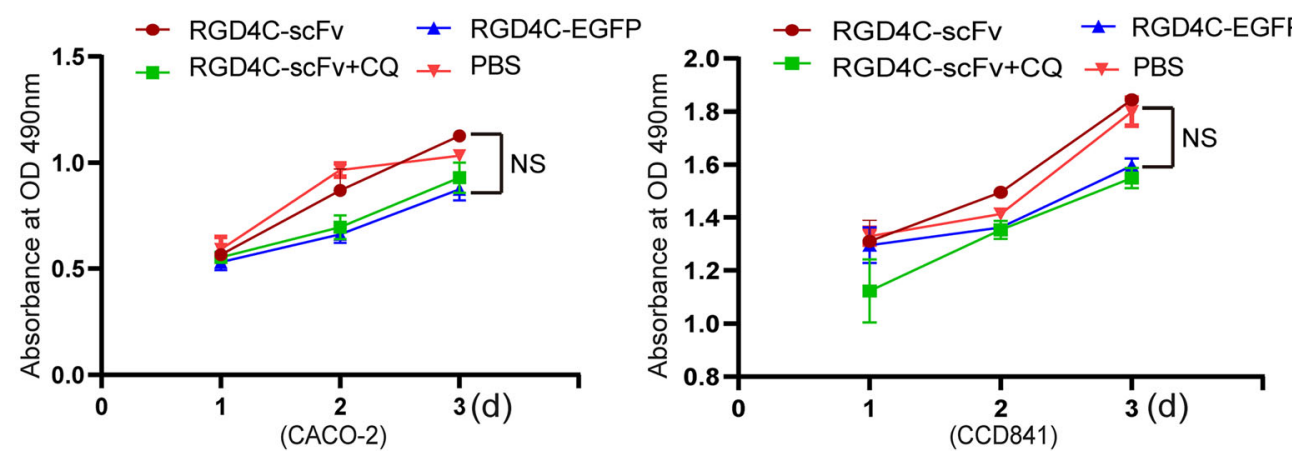

Fig. 5 (See legend on next page.) 
(See figure on previous page.)

Fig. 5 a A colony formation experiment was performed to detect the effect of RGD4C-scFv on SW480 cell proliferation. SW480 cells were incubated with $20 \mu \mathrm{M}$ fusion protein. After 2 weeks of incubation, monoclonal cells were stained with Giemsa. The numbers of tumor cell clones in the RGD4C-scFv and RGD4C-linker-scFv groups were significantly lower than those in the anti-p21Ras scFv and PBS groups. $\mathbf{b}$ The clone numbers of SW480 cell in the RGD4C-sCFv and RGD4C-SCFv+CQ groups were also significantly lower than those in the RGD4C-EGFP and PBS groups. c However, CACO-2 cell clones had no significant difference between the experimental group and the control group. $\mathbf{d}$ The clone numbers of normal cell CCD841 cells in the RGD4C-scFv and RGD4C-scFv+CQ groups were roughly the same with those in the RGD4C-EGFP and PBS groups. e After treatment with RGD4C-p21Ras-scFv for 1d, $2 d$, or $3 d$, the proliferative activity of SW480 cells was tested by an MTT assay. The growth of SW480 cells was inhibited by both RGD4C-scFv and RGD4C-linker-scFv compared with the anti-p21Ras scFv and PBS. f After treatmented with RGD4C-scFv, RGD4C-EGFP or RGD4C-scFv+CQ for 1d, $2 \mathrm{~d}, 3 \mathrm{~d}$, the growth of SW480 cells was inhibited by both RGD4C-scFv or RGD4C-scFv+CQ compared with the RGD4C-EGFP and PBS. $\mathbf{g}$ and $\mathbf{h}$ After treatment with RGD4C-scFv, RGD4C-EGFP or RGD4C-scFv+CQ for 1d, 2 d, 3 d, neither the RGD4C-EGFP and PBS control groups nor the RGD4C-scFv and RGD4C-scFv+CQ experimental group had any killing effect on the CACO-2 and CCD841 cells

\section{Published online: 07 April 2021}

\section{Reference}

1. Huang CC, Liu FR, Feng Q, et al. RGD4C peptide mediates anti-p21Ras sCFy entry into tumor cells and produces an inhibitory effect on the human colon cancer cell line SW480. BMC Cancer. 2021;21:321 https://doi.org/10.11 86/s12885-021-08056-4. 